\title{
EDITOR'S NOTE ON THIS MILESTONE EDITION
}

\author{
GERT ROODT \\ Managing Editor
}

If one looks back at the record of published articles in the $S A$ Journal of Industrial Psychology it becomes evident that the largest majority of articles came from a quantitative and positivist research paradigm. This state of affairs created to a large extent a situation of imbalance of which we will understand the repercussions only at a much later stage.

The quantitative paradigm seeks to explain phenomena from a global perspective, as it tries to unravel the relationships between variables (constructs) by rigorously testing hypotheses to ultimately explain, predict or to control these variables. The research designs normally vary from highly controlled experimental research designs through quasiexperimental designs to ex post facto research designs with very little experimental control. The focus here is on generalisation - to understand the broad picture. This is perhaps why this paradigm appeals to people in managing or governing positions where it is important to have a broad perspective on issues and trends.

The qualitative paradigm on the other hand seeks to understand and to appreciate how people experience certain life events, to interpret and learn how events affect them and also how people adapt and adjust themselves to cope with these events. The focus of this kind of research is inward - on the quality of the experience. Research strategies used in this paradigm are the biography, phenomenology, grounded theory, ethnography and the case study with all kinds of variations within these broad categories. The qualitative paradigm is useful in gaining understanding of phenomena, to build theories or to elaborate on existing theories.

From this short overview of both paradigms it is clear that they are not contradicting one another, nor are they in competition they are merely complementing one another. Human Resource Managers and Industrial Psychologists (as researchers and scientists) need to understand both paradigms and should also be able to command the operational side of these paradigms.

This issue introduces different approaches in the qualitative paradigm and will therefore hopefully act as a reference source for reporting future qualitative research. We trust that this milestone edition under the capable guest editorship of $\mathrm{Dr}$ Willem Schurink will break some of the barriers in the use of qualitative research in the Human Resource Management and in the Industrial Psychology fields. 